\title{
Septic thrombophlebitis with acute osteomyelitis in adolescent children: a report of two cases and review of the literature
}

\author{
Amy A. LePage • Erik P. Hess • Raquel M. Schears
}

Received: 10 January 2008 / Accepted: 5 February 2008/Published online: 10 April 2008

(C) Springer-Verlag London Ltd 2008

\begin{abstract}
The triad of acute osteomyelitis, deep venous thrombophlebitis, and septic pulmonary embolism is a rare, but life-threatening syndrome in children that requires prompt recognition and treatment. We report two cases of acute osteomyelitis complicated by septic thrombophlebitis and pulmonary emboli. Both patients required operative drainage to remove the septic focus. Recognition of any one component of the triad should prompt a search for the other associated disorders. Aggressive management with early antibiotic administration, anticoagulation, and surgical debridement can be life saving.
\end{abstract}

\section{Case reports}

\section{Case 1}

A 13-year-old male presented to the local emergency department (ED) with a 3-day history of worsening left arm pain refractory to non-steroidal anti-inflammatory medications (NSAIDs). Four days prior, he reported participating in a wrestling match. Plain radiographs of the humerus were reportedly negative for any acute bony abnormality, and the patient was discharged on more NSAIDs.

Upon arrival in our emergency department, the patient appeared jaundiced and in moderate distress, complaining of right upper quadrant abdominal pain. Vital signs were

A. A. LePage $\cdot$ E. P. Hess $\cdot$ R. M. Schears $(\bowtie)$

Department of Emergency Medicine, Mayo Clinic,

200 First Street SW,

Rochester, MN 55905, USA

e-mail: schears.rocky@mayo.edu remarkable for a maximal temperature of $38.0^{\circ} \mathrm{C}$ and a sinus tachycardia at 103 beats $/ \mathrm{min}$. Review of systems was positive for nausea. On physical exam, the medial aspect of the left proximal upper extremity was exquisitely tender to palpation from the elbow to the mid-axilla. The right upper quadrant and epigastrium were moderately tender to palpation without guarding or rigidity, and the liver edge was palpable $3 \mathrm{~cm}$ below the costal margin. Laboratory values were remarkable for a $\mathrm{WBC}$ of $5.2 \times 10^{9} / 1$ with $92 \%$ neutrophils, platelet count of $73 \times 10^{9} / 1$, ALT $126 \mathrm{IU} / 1$, AST $133 \mathrm{IU} / 1$, total bilirubin $8.0 \mathrm{mg} / \mathrm{dl}$, and direct bilirubin $5.4 \mathrm{mg} / \mathrm{dl}$. Duplex ultrasound of the left upper extremity revealed occlusive thrombus in the cephalic vein extending from just above the antecubital fossa to the origin of the subclavian vein. Abdominal ultrasound confirmed the suspected hepatosplenomegaly. Chest radiography demonstrated focal infiltrates in the left lung (Fig. 1). Heparin was initially withheld due to the low platelet count and superficial location of the thrombus, and the patient was admitted to the general pediatric ward.

Shortly after admission, the patient became acutely hypoxic, with oxygen saturation of $77 \%$ on room air. He was transferred to the pediatric intensive care unit and stabilized with noninvasive ventilation and intravenous fluids. Emergent CT scan of the chest demonstrated a Yshaped filling defect involving two segmental branches of the right lower lobe indicative of acute pulmonary embolism. The patient was subsequently anticoagulated with low molecular weight heparin and started on broadspectrum antibiotics. Blood cultures grew methicillinsensitive Staphylococcus aureus (MSSA), and antibiotic coverage was adjusted accordingly.

Despite anticoagulation and antibiotic administration, the patient's clinical status failed to improve. On hospital day 5, 


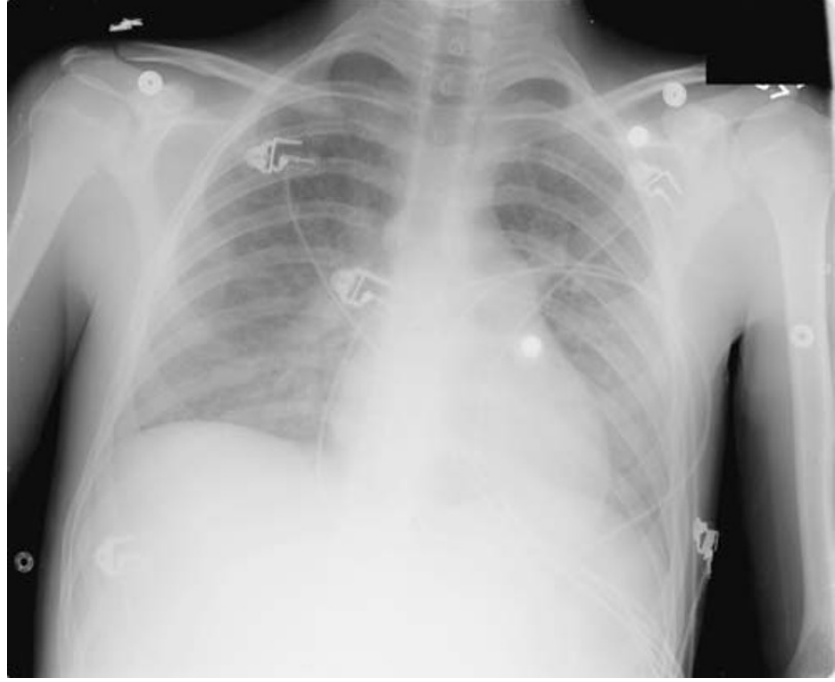

Fig. 1 AP CXR showing mild diffuse bilateral pulmonary infiltrates. $566 \times 465 \mathrm{~mm}(96 \times 96 \mathrm{DPI})$

MRI of the left upper extremity showed a focal area of cortical destruction on the lateral aspect of the proximal humerus with a $2.6 \times 0.8 \times 3-\mathrm{cm}$ fluid collection on the anterior aspect (Fig. 2). The patient underwent operative exploration during which a subperiosteal soft tissue abscess was discovered anterior to the humeral metaphysis. Cultures subsequently grew MSSA. After intravenous antibiotic administration and extensive physical therapy, he was discharged from the hospital in stable condition.

\section{Case 2}

A 15-year-old male presented to the ED complaining of left thigh pain, shortness of breath, and chest discomfort. Two weeks before, he had been seen in an outside ED after sustaining scrapes to his knees after a fall. The initial injury occurred during an indoor ice hockey game, with the abrasions healing over 2 days. At that time, radiographs of the knees were reportedly normal. The patient was dismissed on NSAIDS and crutches for weight-bearing as tolerated. Despite the apparent healing, the left knee continued to hurt, prompting ED re-evaluation. Again the patient was evaluated and discharged, this time with narcotics and non-weight-bearing instructions. Over a few days he worsened, pain confined him to bed, and he developed pleuritic chest pain, low grade fever, chills, a sore throat and a nonproductive cough. His mother brought him to our ED for his third acute care visit in 3 weeks.

Physical examination revealed a tachypneic ill-appearing diaphoretic adolescent male. Vital signs were significant for a temperature of $38.8^{\circ} \mathrm{C}$, pulse of 155 beats $/ \mathrm{min}$, and respiratory rate of 24 breaths/min with room air oxygen saturations of $88 \%$. Bilateral vesicular breath sounds, but no wheezing or crackles were noted on auscultation. His

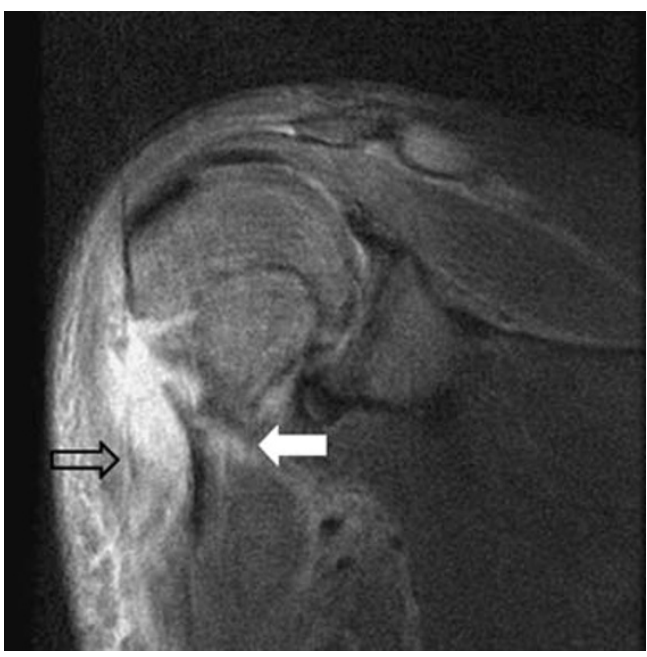

Fig. 2 MRI of the left upper extremity demonstrating focal cortical destruction on the lateral aspect of the proximal humerus (white arrow) and a $2.6 \times 0.8 \times 3-\mathrm{cm}$ fluid collection anteriorly (black arrow); $279 \times 123 \mathrm{~mm}(96 \times 96 \mathrm{DPI})$

peripheral skin exam was normal without rash, swelling, cyanosis, and atraumatic. He did complain of hypesthesias of the left thigh, which limited exam of the thigh, and no crepitus was found. His pulses were present and symmetric in the lower extremities, but capillary refill was decreased, and no signs of veno-occlusive disease were evident.

Laboratory values revealed a WBC of $16.7 \times 10^{9} / 1$, platelet count of $12 \times 10^{9} / 1$, BUN of $64 \mathrm{mg} / \mathrm{dl}$, creatinine of $2.4 \mathrm{mg} / \mathrm{dl}$, and a D-Dimer greater than $2,000 \mathrm{ng} / \mathrm{ml}$. CXR showed mild diffuse bilateral pulmonary infiltrates, and the left hip film demonstrated a vague mottled lucency in the intertrochanteric region (Fig. 3). Duplex ultrasound of the left lower extremity was "negative" for deep vein thrombosis (DVT), but soft tissue changes noted around the left common femoral vein were concerning for subcutaneous air. Bedside transthoracic echo was negative for signs of right ventricular strain. Computed tomography of the chest to exclude pulmonary embolism (PE) was deferred in the setting of acute renal insufficiency. ED differential diagnosis included DIC without rash, heparin-induced thrombocytopenia, pneumonic process or PE versus SIRS secondary to an infectious process of uncertain etiology. The patient was aggressively resuscitated with intravenous fluids, prophylactically anticoagulated and initiated on broad spectrum antibiotics after blood cultures were obtained in the ED. The patient was admitted to the pediatric intensive care unit.

After intravenous fluid rehydration, the patient's creatinine improved to $1.2 \mathrm{mg} / \mathrm{dl}$, and a contrast CT scan of the chest, abdomen, pelvis, and left lower extremity was obtained. Chest CT was negative for PE, but demonstrated diffuse hazy opacification of both lungs with air bronchograms, consistent with ARDS. CT of the left lower 


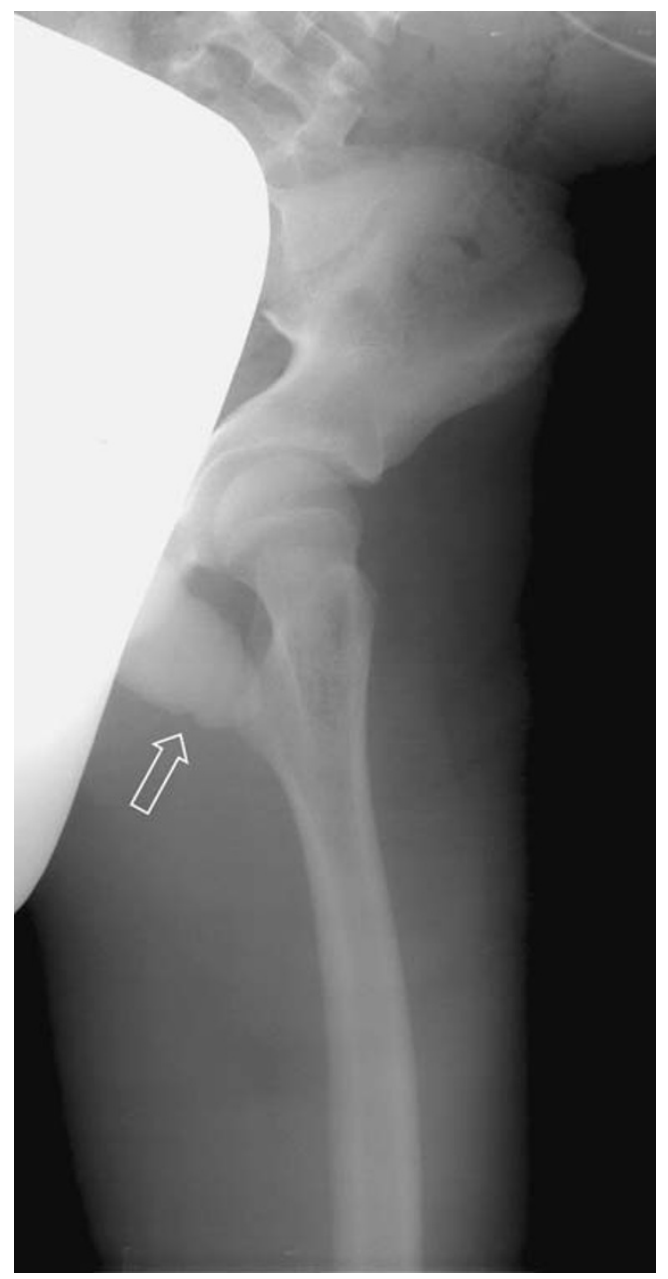

Fig. 3 AP radiograph of the left hip showing a vague, mottled lucency in the intertrochanteric region of the left femur suggestive of intraosseous gas (white arrow); $465 \times 566 \mathrm{~mm}(96 \times 96 \mathrm{DPI})$

extremity demonstrated thrombus and air within the left common femoral vein with fluid throughout the myofascial planes of the left thigh and air within the left femur consistent with osteomyelitis (Fig. 4). Repeat duplex ultrasound of the left lower extremity confirmed thrombus in the left common femoral vein. An inferior vena cava filter was placed to prevent embolization of septic thrombi. The patient subsequently became hypotensive with increasing respiratory distress, requiring inotropic support and mechanical ventilation lasting several days.

After stabilization, MRI of the pelvis and thighs showed extensive thrombosis of the left common femoral vein extending into intramuscular branches of the upper thigh, a $1-\mathrm{cm}$ fluid collection deep within the vastus intermedius, and heterogenous signal within the intramedullary compartment of the shaft and neck of the femur indicative of intraosseous gas secondary to osteomyelitis (Fig. 5).

On hospital day 2, the patient underwent operative irrigation and debridement, with evacuation of a large amount of purulent material followed by serial culture,

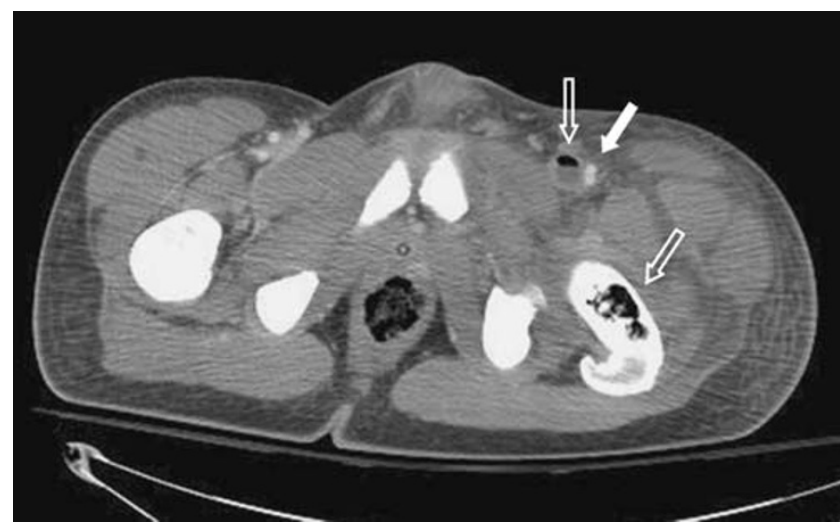

Fig. $4 \mathrm{CT}$ scan of abdomen and pelvis with contrast demonstrating clot (white arrow) and air (gray arrow) within the left common femoral vein and within the left femur consistent with osteomyelitis. Fluid throughout the myofascial planes of the left thigh is also seen; $340 \times 96 \mathrm{~mm}(96 \times 96 \mathrm{DPI})$

incisement, and drainage of affected soft tissues, with limited resection of necrotic bone. Tissue and blood cultures were positive for Fusobacterium necrophorum, an anaerobic gram-negative bacillus. Despite repeated operative irrigation and debridement of the left hip, the patient remained persistently febrile. This occasioned duplex ultrasoundography of the neck and carotids vessels, which failed to reveal evidence of thrombus formation. However, repeat left lower extremity CT demonstrated local thrombus progression necessitating thrombectomy. As thrombus was extracted from segments involving the deep popliteal fossa and distal common iliac vein, concern for central embolization arose again. A repeat chest CT demonstrated small areas of cavitation throughout the dependent infiltrates, suggesting that the infiltrates were at least partially due to septic emboli (Fig. 6). Eventually, the patient recovered

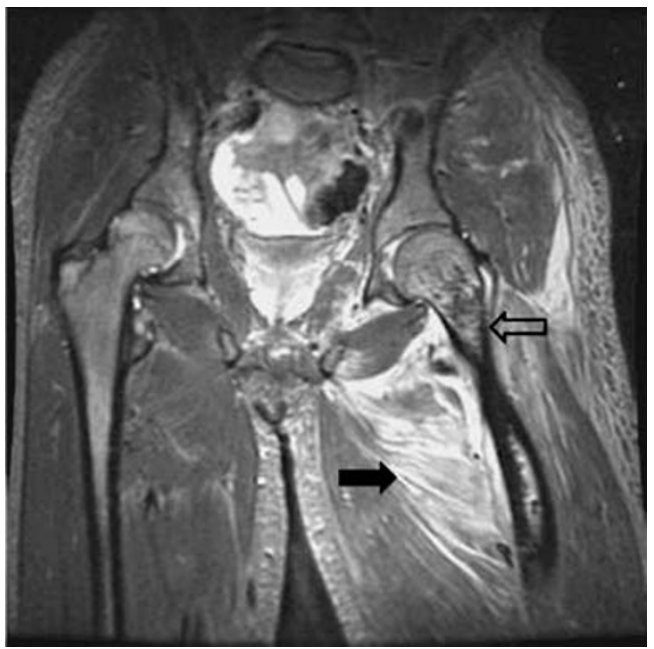

Fig. 5 MRI of the pelvis and thighs extensive soft tissue edema (black arrow) and heterogeneous signal within the intramedullary compartment of the shaft and neck of the femur indicative of intraosseous gas secondary to osteomyelitis (gray arrow) 


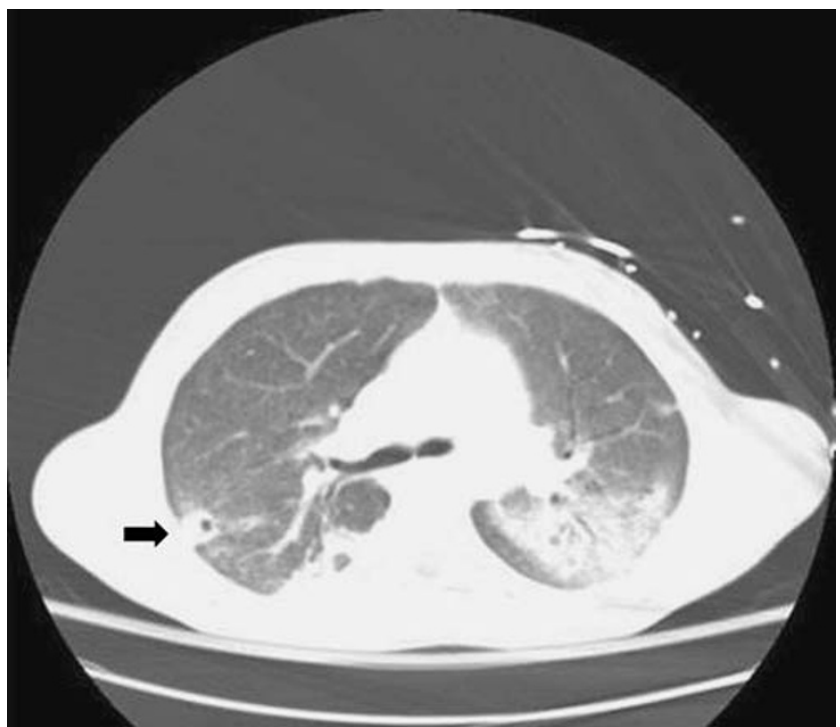

Fig. $6 \mathrm{CT}$ scan of the chest with contrast demonstrating posterior basilar infiltrates and small areas of cavitation in the periphery suggestive of septic emboli (black arrow); 340×96 mm (96×96 DPI)

after prolonged antibiotics and multiple debridement procedures with the involved limb salvaged intact.

\section{Discussion}

Few reports in the literature have emphasized the lifethreatening triad of acute osteomyelitis, deep vein thrombophlebitis, and septic pulmonary embolism. The sequential order in which each component of the triad develops remains controversial [2, 4, 5]. Horvath et al. and Jupiter et al. were among the first to highlight the association of acute osteomyelitis and septic thrombophlebitis in children [4, 5]. The additional association of septic pulmonary embolism was first described by Gorenstein et al. in the context of disseminated staphylococcal infection, and subsequently in a case report by Yuksel et al. [1,2], with a literature review and recommendations for management, more recently, by Nourse et al. [3]. Joint pain out of proportion to exam in a nontoxic, afebrile patient can easily lull the unwary clinician into overlooking early acute osteomyelitis. The delay in diagnosis or bacterial pathogenicity can lead to evolution of deep venous thrombosis, which occurred in both cases presented. Failure to appreciate the propensity of distal deep septic thrombophlebitis to advance to central pulmonary embolic shower can paradoxically create delays in initiation of anticoagulation and may facilitate early hematogenous spread through staged surgical interventions, or potentiate the situation of tardy clot extraction as a result.

Osteomyelitis in children is typically spread hematogenously and occurs most commonly in the long bones of the lower extremities [6, 7]. However, bony infection occurred in the upper extremity in case 1 and in the lower extremity in case 2. In each case, relatively minor trauma had preceded the development of acute osteomyelitis. Our experience coincides with that of Feldman et al. in which infection occurred with equal frequency in both the upper and lower extremities [6]. Typical chest X-ray findings early in the course of combined pulmonary and osteal infection consist of multiple poorly defined densities predominantly in the peripheral and inferior segments of the lung. These findings were not well discriminated by chest X-rays in our two patients.

In acute osteomyelitis secondary to $S$. aureus, the leukocyte count is typically normal, although associated with a left shift [8]. This was observed in case 1, in which the causative organism was MSSA. Although a leukocyte count of $16.7 \times 10^{9} / 1$ with a left shift was observed in case 2 , infection in this case occurred secondary to Fusobacterium necrophorum. The F. necrophorum bacteria is a commensal organism of the oropharynx commonly associated with Lemierre's syndrome, which is a disorder characterized by acute oropharyngeal infection with secondary septic thrombophlebitis of the internal jugular vein and metastatic infection to the lungs and joints [9]. Septic pulmonary embolism has also been described with this syndrome [10]. Though not a primary complaint, the patient in case $2 \mathrm{com}$ plained of a sore throat in addition to a nonproductive cough, fever, and chills. Acute pharyngeal infection may have contributed to the subsequent development of septic thrombophlebitis and septic pulmonary embolism. However, no evidence of neck vein thrombophlebitis was demonstrated in this patient.

In conclusion, the triad of acute osteomyelitis, deep venous thrombophlebitis, and septic pulmonary embolism is a rare, but life-threatening syndrome in adolescents that requires prompt recognition and treatment. Detection of any single component of the triad should prompt a search for the other associated disorders. Thromboembolic burden may need to be reassessed frequently if the status of the patient worsens during supportive intensive care hospitalization. Aggressive intervention with early antibiotic administration, anticoagulation, and surgical consultation for intervention started in the ED can be life saving.

\section{References}

1. Yuksel H, Ozguven AA, Akil I, Erguder I, Yilmaz D, Cabuk M (2004) Septic pulmonary emboli presenting with deep venous thrombosis secondary to acute osteomyelitis. Pediatr Int 46:621623

2. Gorenstein A, Gross E, Houri S, Gewirts G, Katz S (2000) The pivotal role of deep vein thrombophlebitis in the development of acute disseminated staphylococcal disease in children. Pediatrics 106:E87 
3. Nourse C, Starr M, Munckhof W (2007) Community-acquired methicillin-resistant Staphylococcus aureus causes severe disseminated infection and deep venous thrombosis in children: literature review and recommendations for management. J Paediatr Child Health 43(10):656-661, Oct

4. Horvath FL, Brodeur AE, Cherry JD (1971) Deep thrombophlebitis associated with acute osteomyelitis. J Pediatr 79:815-818

5. Jupiter JB, Ehrlich MG, Novelline RA, Leeds HC, Keim D (1982) The association of septic thrombophlebitis with subperiosteal abscesses in children. J Pediatr 101:690-695

6. Wall EJ (1998) Childhood osteomyelitis and septic arthritis. Curr Opin Pediatr 10:73-76
7. Felman AH, Shulman ST (1975) Staphylococcal osteomyelitis, sepsis and pulmonary disease. Observations of 10 patients with combined osseous and pulmonary infections. Radiology 117:649-655

8. Hollmig ST, Copley LA, Browne RH, Grande LM, Wilson PL (2007) Deep venous thrombosis associated with osteomyelitis in children. J Bone Joint Surg Am 89(7):1517-1523, Jul

9. Karkos PD, Karkanevatos A, Panagea S, Dingle A, Davies J (2004) Lemierre's syndrome: how a sore throat can end in disaster. Eur J Emerg Med 4:228-230

10. Gormus N, Durgut K, Ozergin U, Odev K, Solak H (2004) Lemierre's syndrome associated with septic pulmonary embolism: a case report. Ann Vasc Surg 18(2):243-245, Mar 